\title{
The impact of the introduction of fidaxomicin on the management of Clostridium difficile infection in seven NHS secondary care hospitals in England: a series of local service evaluations
}

\author{
S. D. Goldenberg ${ }^{1} \cdot$ S. Brown ${ }^{2}$ L. Edwards ${ }^{3}$ - D. Gnanarajah ${ }^{4} \cdot$ P. Howard $^{5}$.

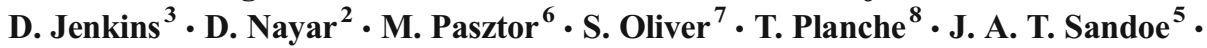 \\ P. Wade ${ }^{1} \cdot$ L. Whitney ${ }^{8}$
}

Received: 14 August 2015 / Accepted: 9 November 2015 /Published online: 12 December 2015

(C) The Author(s) 2015. This article is published with open access at Springerlink.com

\begin{abstract}
Clostridium difficile infection (CDI) is associated with high mortality. Reducing incidence is a priority for patients, clinicians, the National Health Service (NHS) and Public Health England alike. In June 2012, fidaxomicin (FDX) was launched for the treatment of adults with CDI. The objective of this evaluation was to collect robust real-world data to understand the effectiveness of FDX in routine practice. In seven hospitals introducing FDX between July 2012 and July 2013, data were collected retrospectively from medical records on CDI episodes occurring 12 months before/after the introduction of FDX. All hospitalised patients aged $\geq 18$ years with primary $\mathrm{CDI}$ (diarrhoea with presence of toxin $\mathrm{A} / \mathrm{B}$ without a previous CDI in the previous 3 months) were included. Recurrence was defined as in-patient diarrhoea re-emergence requiring treatment any time within 3 months after the first
\end{abstract}

\footnotetext{
S. D. Goldenberg

Simon.Goldenberg@gstt.nhs.uk
}

1 Centre for Clinical Infection and Diagnostics Research, King's College, London and Guy's and St Thomas' NHS Foundation Trust, 5th Floor, North Wing, St Thomas' Hospital, Westminster Bridge Road, London SE1 7EH, UK

2 Department of Microbiology, County Durham and Darlington NHS Foundation Trust, Durham, UK

3 University Hospitals of Leicester NHS Trust, Leicester, UK

4 Derby Hospitals NHS Foundation Trust, Derby, UK

5 Department of Microbiology, University of Leeds and Leeds Teaching Hospitals NHS Trust, Leeds, UK

6 University Hospitals of Morecambe Bay NHS Foundation Trust, Lancaster, UK

7 pH Associates Ltd, Marlow, UK

8 St George's Healthcare NHS Trust, London, UK episode. Each hospital had a different protocol for the use of FDX. In hospitals A and B, where FDX was used first line for all primary and recurrent episodes, the recurrence rate reduced from $10.6 \%$ to $3.1 \%$ and from $16.3 \%$ to $3.1 \%$, with a significant difference in 28-day mortality from $18.2 \%$ to $3.1 \%(p<0.05)$ and $17.3 \%$ to $6.3 \%(p<0.05)$ for hospitals $\mathrm{A}$ and $\mathrm{B}$, respectively. In hospitals using FDX in selected patients only, the changes in recurrence rates and mortality were less marked. The pattern of adoption of FDX appears to affect its impact on CDI outcome, with maximum reduction in recurrence and all-cause mortality where it is used as firstline treatment.

\section{Introduction}

Clostridium difficile infection (CDI) is a major public health concern [1] and is associated with prolonged hospital stay [2, $3]$, hospital readmissions $[4,5]$ and increased mortality [6-8]. Recurrence of disease is a significant problem, with $20-30 \%$ of patients experiencing a recurrent episode following initial resolution $[9,10]$. The European Society of Clinical Microbiology and Infectious Diseases (ESCMID) guidelines recognise that the high recurrence rate is among the most important problems posed by CDI [11]. In 2012, fidaxomicin (FDX) became available in the UK for the treatment of CDI. Two double-blind, randomised controlled trials (RCTs) showed non-inferiority of FDX compared to vancomycin with respect to initial CDI cure rates, and significantly lower recurrence rates in patients treated with FDX [12, 13]. Data on the realworld use of FDX outside of a clinical trial setting are lacking.

We report a series of local service evaluations conducted to evaluate the impact of FDX introduction on service delivery and CDI management in real-world conditions. Although 
conducted primarily to inform local decision-making, the results of the evaluations reported here detail differences in the experiences of routine FDX use in the English National Health Service (NHS).

\section{Materials and methods}

A series of local service evaluations was conducted between December 2013 and October 2014 in collaboration with microbiologists, infection control clinicians and pharmacists in seven NHS hospitals in England, each offering a range of services, including: secondary, tertiary, acute, long-stay, elective and non-elective care. The primary objective of each evaluation was to describe CDI recurrence rates occurring in the year before (pre-) and after (post-) local introduction of FDX. Secondary objectives included describing: time to clinical cure of CDI; 28day and 3-month all-cause mortality; CDI-related treatment pathways (including times from symptom onset to stool sampling, diagnosis and symptom resolution) and resource use [hospital length of stay (LOS), intensive care unit (ICU) admission rate and LOS, duration of treatment, readmission rate and LOS for both primary and recurrent episodes] in the year before and after FDX introduction; and characteristics of patients receiving FDX.

Hospitals were invited to conduct an evaluation based on interest in describing the impact of local FDX introduction and the availability of at least 20 cases of CDI per year to allow the generation of meaningful results locally. All hospitals conducting an evaluation commenced FDX use between July 2012 and July 2013.

Service evaluations are exempt from ethical review and patient consent in the UK [14]. Each hospital approved the evaluation protocol and release of fully anonymised patient data to $\mathrm{pH}$ Associates Ltd, a research consultancy company, for analysis.

Each evaluation was conducted according to a common protocol. Primary CDI was defined as diarrhoea with $>3$ consecutive unformed bowel movements and presence of $C$. difficile toxin $\mathrm{A} / \mathrm{B}$ in stool (see Table 1 for the testing protocols). Two different definitions of CDI recurrence were used to accommodate local differences in repeating $C$. difficile toxin testing within 28 days of the primary infection. In hospitals A, C and G, policy was to define recurrence as symptomatic (i.e. reappearance of diarrhoea to an extent, judged by the frequency of passed unformed stools, that was greater than the frequency noted on the last day of medication) with treatment for CDI and toxin confirmed. In hospitals B, D, E and F, policy was to assume recurrence if patients were symptomatic and given treatment for CDI, without a requirement for toxin testing. CDI was considered to be community-acquired if the symptoms and/or sample for toxin testing were documented within $48 \mathrm{~h}$ of hospital admission.

Recurrences presenting to the same hospital within 3 months of the primary CDI episode (first toxin-positive stool sample) were recorded for the evaluation. Clinical cure was defined as three or fewer unformed stools on two consecutive

Table 1 Clostridium difficile toxin testing policies and C. difficile infection (CDI) severity criteria

\begin{tabular}{|c|c|c|}
\hline Hospital & C. difficile toxin testing policy & CDI severity criteria \\
\hline A & $\begin{array}{l}\text { Two-step testing method: } 1 \text {. glutamate dehydrogenase } \\
\text { (GDH) enzyme immunoassay (EIA); } 2 \text {. C. difficile toxin EIA }\end{array}$ & According to the ESCMID definitions \\
\hline B & Two-step testing method: 1 . GDH EIA; 2. C. difficile toxin EIA & Severity parameters not collected for this evaluation \\
\hline $\mathrm{C}$ & Two-step testing method: 1 . GDH EIA; 2. C. difficile cytotoxin assay & $\begin{array}{l}\text { Life-threatening: any of: hypotension (not responsive } \\
\text { to fluid challenge), partial or complete ileus or toxic } \\
\text { megacolon } \\
\text { Severe: any of: raised WCC }>15 \times 10^{9} / \mathrm{L} \text {., acute kidney } \\
\text { injury (e.g. }>50 \% \text { rise in serum creatinine above baseline) } \\
\text { temperature of }>38.5^{\circ} \mathrm{C} \text {, evidence of severe colitis } \\
\text { (e.g. guarding, abdominal tenderness } \\
\text { Non-severe: diarrhoea without features of severe } \\
\text { or life-threatening infection }\end{array}$ \\
\hline $\mathrm{D}$ & Two-step testing method: 1 . GDH EIA; 2. C. difficile toxin EIA & According to PHE guidelines \\
\hline $\mathrm{E}$ & $\begin{array}{l}\text { Three-step testing method: } 1 \text {. screen with GDH EIA; } \\
\text { 2. all GDH-positives are then tested for } C \text {.difficile toxin EIA; } \\
\text { 3. if GDH-positive and toxin-negative, PCR testing }\end{array}$ & According to PHE guidelines \\
\hline $\mathrm{F}$ & $\begin{array}{l}\text { Two-step testing method: } 1 \text {. GDH EIA; } 2 \text {. C. difficile } \\
\text { toxin EIA } \\
\text { GDH-positive, toxin-negative has another stool } \\
\text { sample tested at least } 24 \mathrm{~h} \text { later }\end{array}$ & According to PHE guidelines \\
\hline G & $\begin{array}{l}\text { Three-step testing method: } 1 \text {. screen with GDH EIA; } \\
\text { 2. all GDH-positives are tested for } C \text {. difficile toxin EIA, } \\
\text { 3. if GDH-positive and toxin-negative, PCR testing }\end{array}$ & According to PHE guidelines \\
\hline
\end{tabular}


days following treatment. The date of diarrhoea resolution was noted as recorded in clinical records; if this was unavailable, the date diarrhoea was last documented or the stop date of CDI medication in the clinical record was noted. Severity of CDI could not be defined objectively by retrospective review and was recorded as documented in the clinical record.

Each evaluation included two 12-month retrospective observation periods for primary CDI episodes, one before and one following local introduction of FDX. To ensure identification of all recurrences within 3 months of a primary episode, an additional 3-month observation period was included at the end of each observation period, for the collection of recurrence data only (Fig. 1). Similarly, microbiology records for the previous 3 months were checked for all CDI infections to verify a primary rather than recurrent episode.

All patients with a positive toxin A/B test for $C$. difficile within the evaluation period (pre- or post-FDX) were identified from microbiology department records. Patients aged $<18$ years were excluded, as were any cases that were diagnosed and managed in a community setting without hospital admission.

The full evaluation data were obtained for all episodes in the pre/post periods in all hospitals except hospital C. In this hospital, all episodes were included in the recurrence rate endpoint (Table 4, row 1) but the full dataset was collected in only a sample of patients in order to manage numbers (all other tables). The sample was taken by selecting the first seven episodes per month during the pre period and all electronically available episodes in the post period, both methods generating a random sample. Data were collected retrospectively from patients' hospital records, including paper notes and electronic databases as appropriate in each hospital, using a standard data extraction process. Data were fully anonymised for analysis. The evaluation relied on data recorded routinely for patients' clinical care. Analyses were conducted using the available data, with the number of patients included in each analysis stated where data were missing. Patients who died whilst inpatients were excluded from LOS calculations.

FDX was adopted for different uses in each hospital, with some using the agent first line in all patients (primary and recurrent $\mathrm{CDI}$ ), others only for $\mathrm{CDI}$ recurrence and others only in selected patients (Table 2). In order to appreciate the response to different patterns of use, the results of each evaluation are presented separately, grouped according to the local policy for the use of FDX.

Values for each measure were tabulated and examined visually for possible differences between treatments (pre- and post-FDX) within each hospital. Differences between percentages or rates were tested for statistical significance using the Chi-squared $\left(\chi^{2}\right)$ test, except when the total number of observations was too small and Fisher's exact test was used. Continuous variables were compared using the unpaired $t$-test or Mann-Whitney $U$-test, depending on data distributions and homogeneity of the variance.

\section{Results}

The FDX prescribing policy at each hospital is shown in Table 2. Following the introduction of FDX, hospitals A and $\mathrm{B}$ used FDX for all patients and hospital D used FDX first line in the single CDI recurrence but not for most primary CDI episodes. Hospitals C, E, F and G used FDX first line only in selected patients (primary and recurrent CDI).

The patient characteristics are shown in Table 3 . The median age at primary and recurrent CDI ranged from 70 to 81 years and 43.5 to 84.5 years, respectively. Where severity was documented, the proportion of patients with primary CDI documented as severe/complicated ranged from $7.4 \%$ to $72.2 \%$. Where documented, prior antibiotic use (within 3 months) ranged from $74.1 \%$ to $98.1 \%$ and proton pump inhibitor use concurrent
Fig. 1 Service evaluation design showing two retrospective observation periods, before (pre) and after (post) introduction of fidaxomicin (FDX)

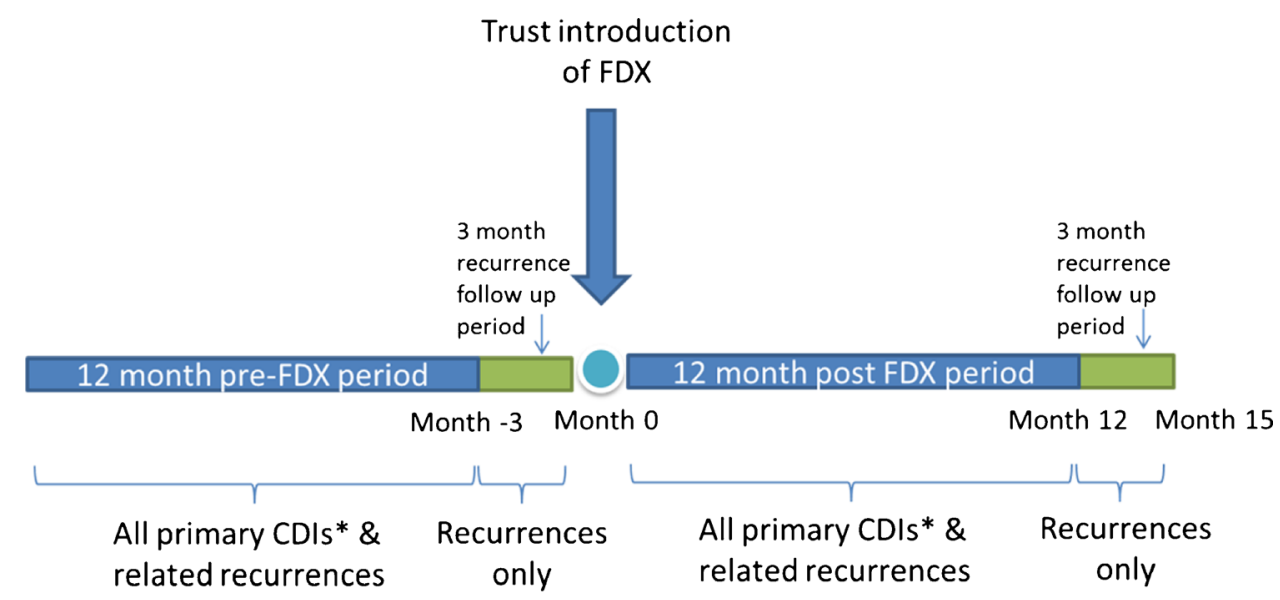

* primary CDI confirmed by checking for CDIs in previous 3 months 


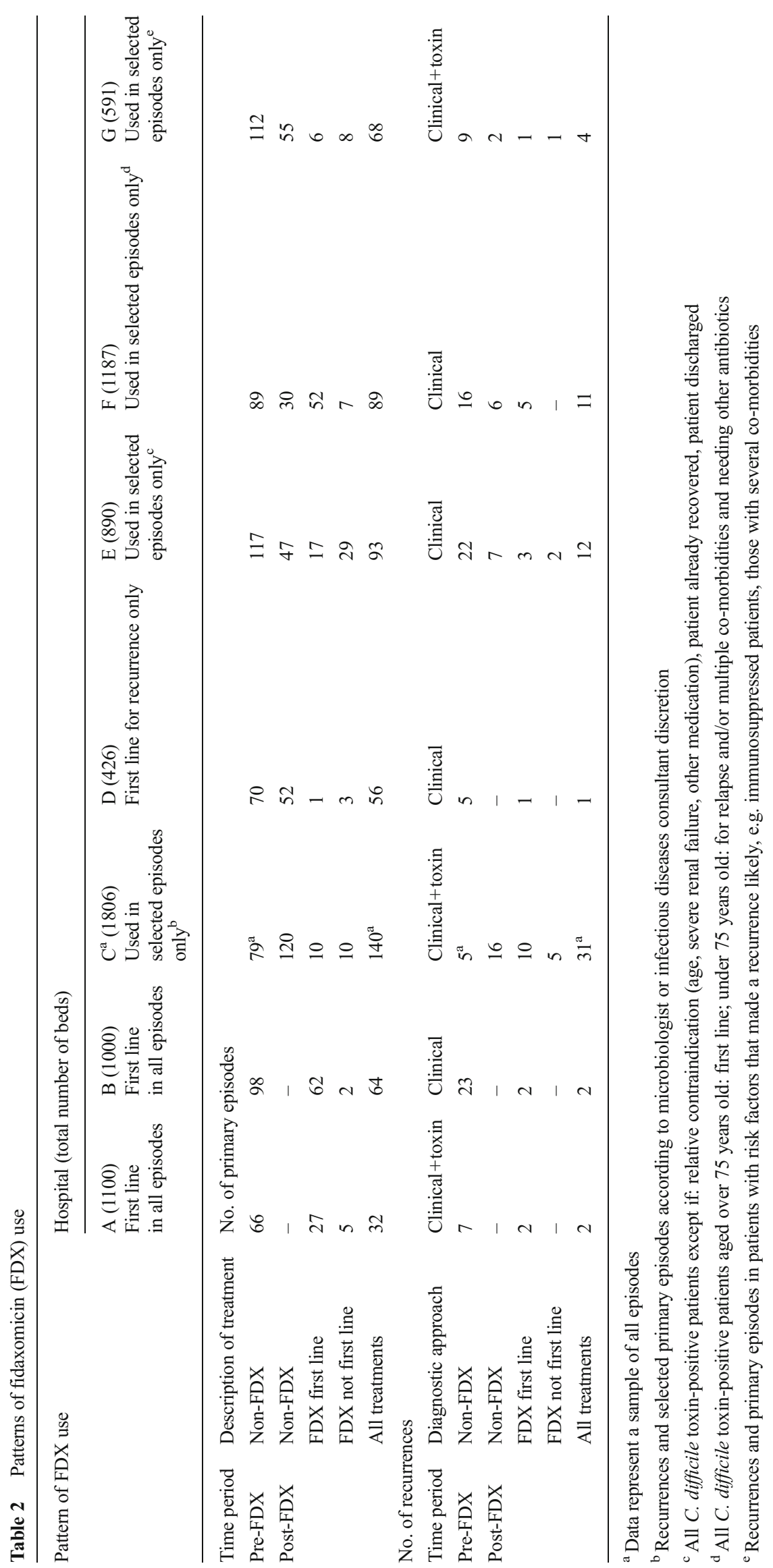




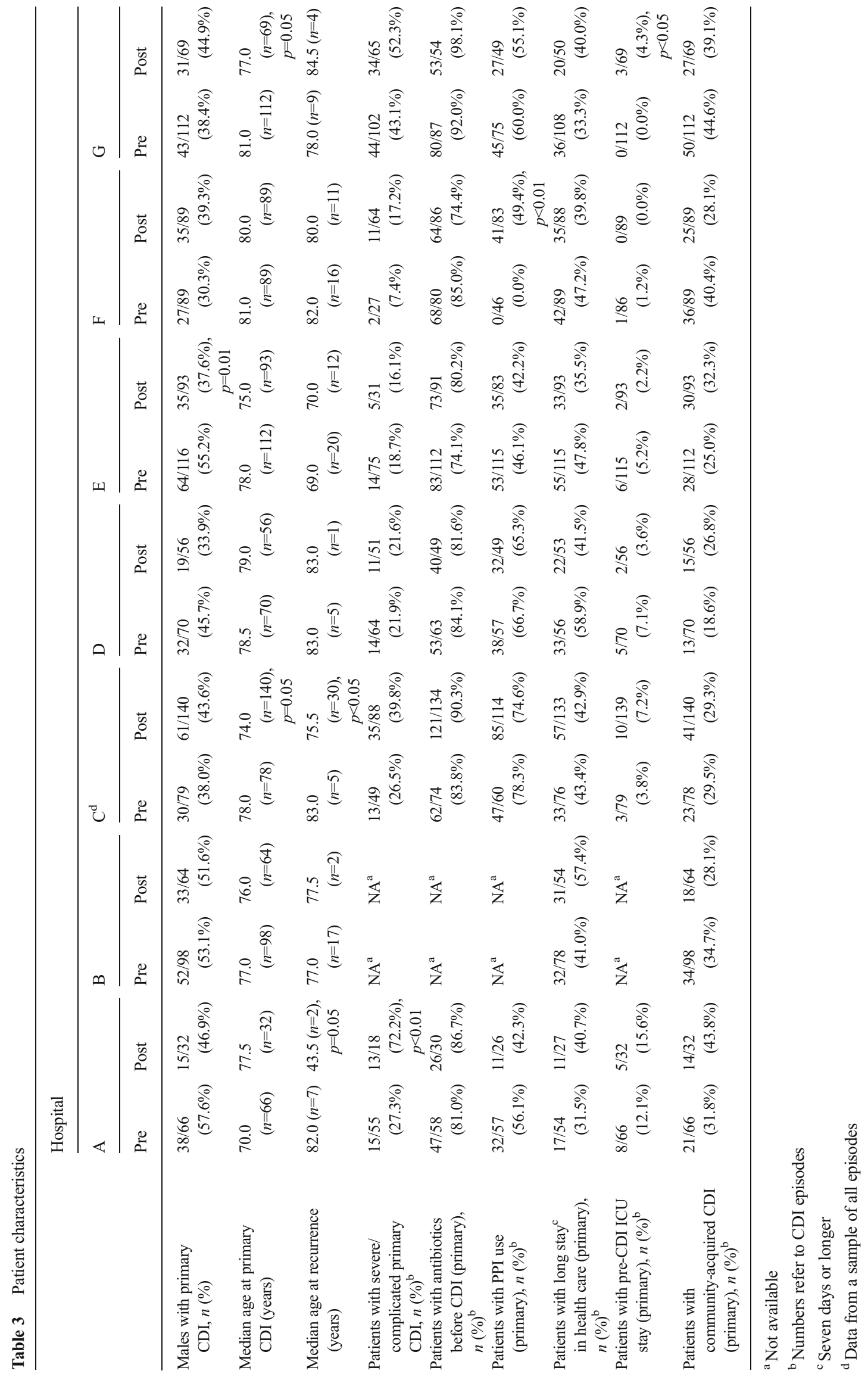


with symptom onset from $0 \%$ to $78.3 \%$. The proportion of patients hospitalised for $\geq 7$ days before CDI ranged from $31.5 \%$ to $58.9 \%$ and the proportion with a prior stay in the ICU during the current hospital admission ranged from $0 \%$ to $12.1 \%$. The proportion of community-acquired primary CDI ranged from $18.6 \%$ to $44.6 \%$.

Table 4 shows the clinical outcomes at each hospital in the pre- and post-FDX periods. The recurrence rate from primary episodes of CDI significantly reduced in the post-FDX period in hospital B by $81.0 \%$ ( $p=0.01$, see Fig. 2$)$. Hospitals A, D, $\mathrm{E}$ and $\mathrm{F}$ had smaller, non-significant, relative reductions in recurrence rates and hospitals $\mathrm{C}$ and $\mathrm{G}$ had small nonsignificant relative increases in recurrence rates.

Also shown in Table 4, the median time from diagnosis of primary CDI to diarrhoea resolution in the post-FDX period increased significantly in hospitals A and C, but remained similar in other hospitals. The median LOS for the primary CDI increased in the post-FDX period in hospital A $(p=0.01)$ and non-significantly in hospital B $(p=0.06)$, but remained similar in other hospitals. The readmission rate within 30 days of discharge after the primary CDI reduced in the post-FDX period in hospital A $(p<0.05)$. There were no significant changes in any other hospital. The median LOS for recurrent CDI episodes showed no significant difference in any hospital between the pre-/post-FDX periods, although the number of recurrences was small. Rates of readmission within 30 days following recurrences showed a non-significant reduction in the post-FDX period in all five hospitals providing this data, although the numbers were small. All-cause mortality within 28 days of primary episodes reduced significantly in the post-FDX period in hospitals $\mathrm{A}, \mathrm{B}$ and $\mathrm{D}$, reduced non-significantly in hospitals $\mathrm{C}, \mathrm{E}$ and $\mathrm{G}$, and non-significantly increased in hospital $\mathrm{F}$ (Table 4).

No adverse events attributed to FDX had been documented in the medical records of any of the patients included in these evaluations.

\section{Discussion}

This series of local service evaluations was conducted primarily for local use but, considered together, the results provide some useful insights on the real-world impact of different FDX adoption strategies.

As expected, patients with CDI were predominantly elderly, with a median age of at least 70 years across the hospitals. The severity of primary CDI was not recorded for all patients in some hospitals, providing an incomplete picture. The wide variation between hospitals in the proportion of patients with severe/complicated CDI also suggests that severity may not be assessed according to the same criteria in different hospitals. Variation in the proportion of patients with communityacquired CDI between hospitals could be partly a reflection of local outbreaks. It would be interesting to understand the impact of FDX on different ribotypes of $C$. difficile in the real world; however, this information was not and is not routinely documented in the normal practice of these hospitals; it may be the subject for potential further study with a prospective design.

Pre-FDX recurrence rates varied between hospitals (5.4 $21.1 \%$ ) but were generally lower than commonly quoted expected recurrence rates of $20-30 \%[15,16]$. It is difficult to compare the results of RCTs with real-world evaluations (RWE) because RCTs generally include highly selected patients in a controlled environment, with strict monitoring, while RWE do not; hence, the difference is most likely to be due to less rigorous surveillance for returning symptoms, and different testing and reporting requirements for recurrences outside an RCT.

The greatest reduction in relative recurrence rates $(>70 \%)$ was observed in the two hospitals that used FDX as first line in all patients with CDI. RCTs showed that FDX reduced recurrence rates compared with vancomycin $[12,13]$ and, therefore, the reduced recurrence in hospitals A and B are consistent with expectations, since all patients were treated with FDX in the post-FDX period. In contrast, the hospitals using FDX more selectively (e.g. only in the elderly or those with severe CDI) observed more modest reductions in relative recurrence rates. Conversely, two hospitals where FDX was used most selectively (chiefly for recurrences only) observed a non-significant increase in recurrence rates.

These data suggest that FDX is most effective when used in all patients with CDI, with no clear indication of patient subgroups most likely to benefit. However, the recurrence rates in all hospitals, irrespective of the pattern of adoption of FDX (3.1-12.5\%), were lower than those seen in the pivotal clinical trials $(12.7-15.4 \%)[12,13]$, despite a shorter window for recording recurrence in the trials (30 days) compared to the service evaluations ( 3 months). This may be explained by the implementation of other infection control measures in the intervening period or changing patterns of ribotypes causing disease and/or the follow-up for recurrence in these evaluations only during the hospital admission and any readmissions, missing CDI recurrences diagnosed in the community.

The time to resolution of diarrhoea was not positively affected by the introduction of FDX in most hospitals and, in fact, there was a statistically significant increase from 7.5 to 13.0 days in hospital A and from 9 to 10 days in hospital C. However, the resolution of symptoms was not well documented in patients' clinical notes, with proxy measures for symptom resolution collected in the absence of definitive documentation, making it difficult to infer the impact of FDX on symptom resolution.

The LOS for primary and recurrent episodes of CDI was not significantly affected by the introduction of FDX in any hospitals, and, in fact, increased in all except one hospital. The factors affecting the LOS remain unclear but may reflect the wellrecognised challenge of discharging patients (particularly the elderly) from hospital [17-19]. The increase in LOS was most marked in hospitals A and B. These hospitals also demonstrated 


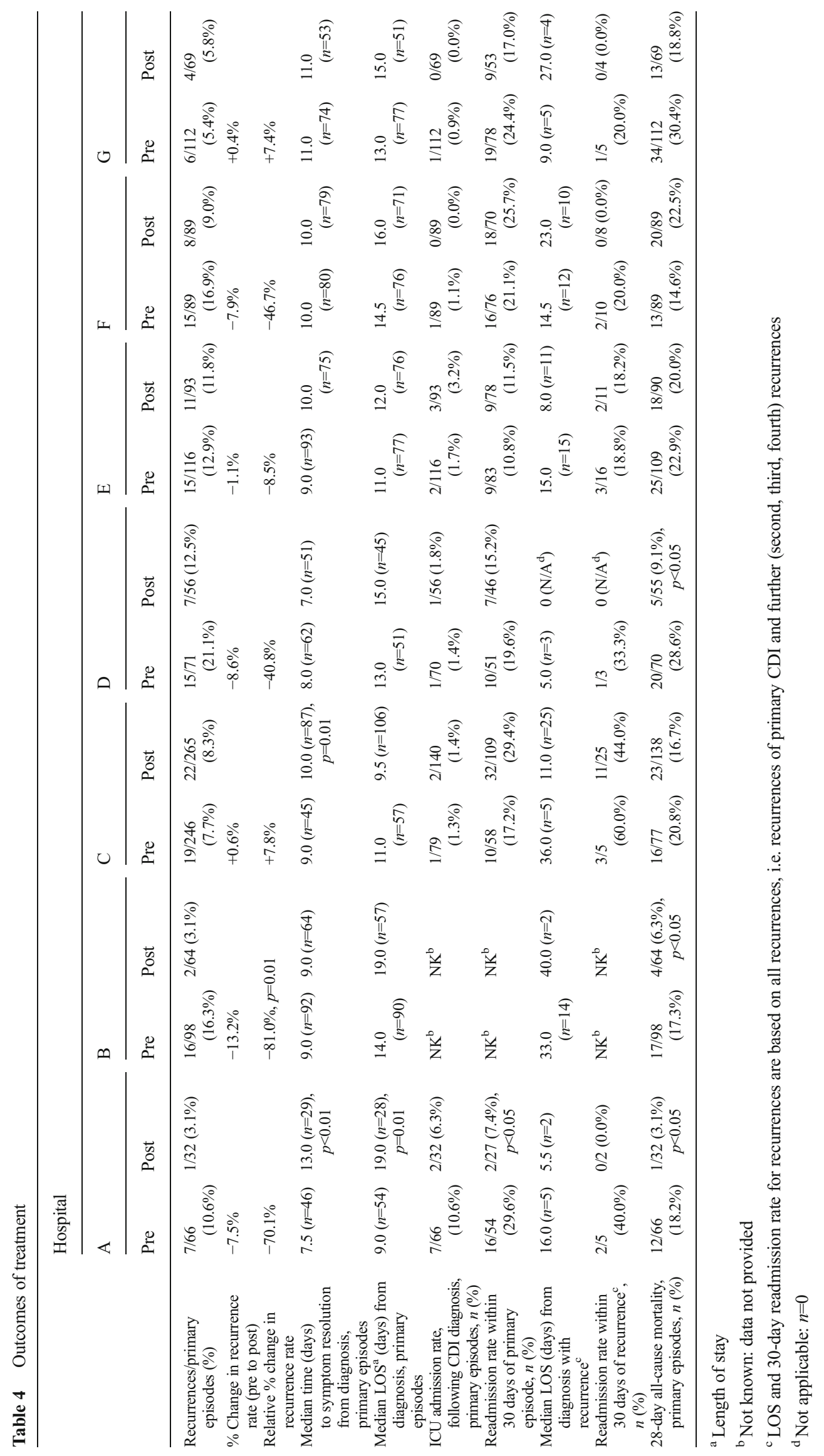




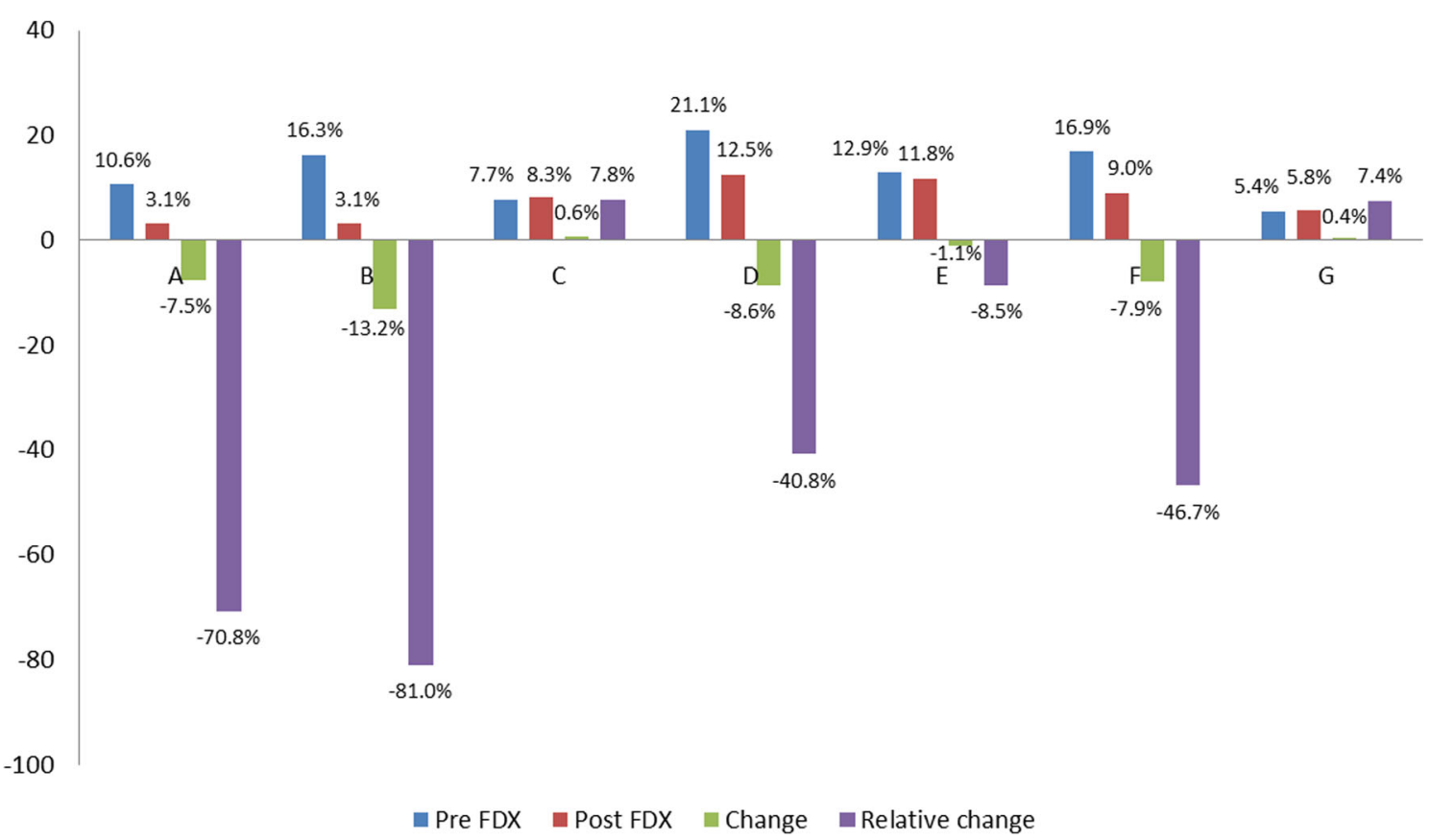

Fig. 2 Clostridium difficile infection (CDI) recurrence rates in the pre- and post-FDX observation periods at participating hospitals

a significant reduction in 28-day mortality in the post-FDX period. Whilst purely speculative, it is possible that the increased LOS may be related to improved survival, with patients who might previously have died and been excluded from the LOS analysis in the pre-FDX period now surviving but requiring extended care.

Mortality rates (28-day all-cause) following primary episodes of CDI were reduced to the lowest levels in hospitals A and B, where FDX was used first line in all patients, although there was also a statistically significant reduction in hospital D, where FDX was only used first line for CDI recurrences.

Readmission within 30 days of the primary or recurrent episode was not consistently affected by the introduction of FDX. Only hospital A, where FDX was introduced for all episodes of CDI, observed a statistically significant reduction in the 30-day readmission rate following primary CDI episodes, although a contributor to this could be the relatively long average LOS among the patients with CDI at this hospital, which may mitigate against readmission. Nevertheless, this reduction is consistent with the significant reduction in the recurrence rate (readmission data were not available for hospital B, which also demonstrated a substantial difference in recurrence).

\section{Limitations}

Some limitations of this series of service evaluations have been described already in the discussion of each outcome measure. In addition, the differing retest policies of hospitals affected our ability to quantify the recurrence rate consistently across all the evaluations, by positive toxin test result, i.e. different definitions of 'recurrence' were used between hospitals and, so, the absolute recurrence rates should not be compared between hospitals. However, definitions of recurrence were consistent over time at each hospital, so the pre-FDX vs. post-FDX comparisons for each hospital are valid. The time to recurrence according to toxin result was affected by each hospital's retest policy; no toxin-positive recurrence could occur within 14 (or 28) days of primary CDI in the hospitals with this policy; hence, this cannot be compared between hospitals. The time to recurrence analysis was also affected by widespread missing dates of recurrence. The definition of resolution of symptoms was not well defined and prone to great variation between hospitals. In the hospitals where toxin positivity was required for the diagnosis of a recurrence, the LOS associated with primary CDI could be an over-estimate due to their criteria for the diagnosis of recurrence. Some hospitals did not collect, or not consistently, data on readmissions. This, again, limited us to comparing between pre- and post-FDX introduction within each hospital, rather than comparing outcomes between hospitals. In hospital C, the selection of a sample of patients for collection of the full study dataset introduces the possibility of bias compared to the other hospitals where all episodes of CDI were included. However, random sampling should have minimised this risk.

\section{Conclusion}

Although not designed as a formal comparison between hospitals, the results of these service evaluations suggest that the pattern of adoption of fidaxomicin (FDX) may affect the overall outcomes of Clostridium difficile infection (CDI) treatment 
achieved by a hospital. In practice, the benefits of the superior effect of FDX on the recurrence rate and 28-day mortality may be realised to the greatest extent where FDX is used as the routine first-line option for the treatment of CDI rather than in selected patients only. A policy of selective use of FDX only for recurrences had good outcomes in one hospital but was based on a small number of treated cases and requires a larger sample size for confirmation.

Acknowledgements The authors gratefully acknowledge the assistance of F. Percival, $\mathrm{pH}$ Associates Ltd, for medical writing assistance in the preparation and editing of this manuscript. $\mathrm{pH}$ Associates Ltd also provided support to Astellas UK and the authors in the study design, conduct of this research, analysis of the results, and review and approval of this manuscript.

\section{Compliance with ethical standards}

Funding This study was funded by Astellas UK Ltd, who participated in the study design, interpretation of data and review of this manuscript. The authors retain the final control of the content.

Conflict of interest In the past 3 years, SDG has received speakers fees from Astellas, BD, Luminex Corp, Orion Diagnostica; consultancy fees from Abbott, Alcimed, Aquarius Population Health, Astellas, GLG Research, Merck, Nicox, pH associates, Qiagen; research grants from Astellas, Enigma Diagnostics, Bio-Rad, GenMark, Luminex Corp; employment from Westminster Clinical Infection Consultants LLP.

TP has provided advice to Astellas, Pfizer, Summit, Cepheid, Launch, Royal College of Physicians and UK Department of Health, and has been an invited speaker of Astellas, Focus, Cepheid and Becton Dickinson.

$\mathrm{PH}$ has received research grants from Astellas, B. Braun, Eumedica, Novartis; speakers honoraria from Astellas, AstraZeneca, B. Braun, Gilead, Pfizer; advisory board honoraria from Astellas, Baxter, B. Braun, Cubist, Gilead, MSD, Pfizer.

$\mathrm{SO}$ is an employee of $\mathrm{pH}$ Associates, who were commissioned by Astellas to provide the support indicated above.

PW, MP, LW, DN, SB and JATS have received advisory board fees from Astellas. DJ has received payments for speaker fees and advisory boards from Astellas.

LE and DG have no conflict of interest to declare.

Open Access This article is distributed under the terms of the Creative Commons Attribution 4.0 International License (http:// creativecommons.org/licenses/by/4.0/), which permits unrestricted use, distribution, and reproduction in any medium, provided you give appropriate credit to the original author(s) and the source, provide a link to the Creative Commons license, and indicate if changes were made.

\section{References}

1. Aguado JM, Anttila VJ, Galperine T et al; CDI Consensus Consortium (2015) Highlighting clinical needs in Clostridium difficile infection: the views of European healthcare professionals at the front line. J Hosp Infect 90:117-125
2. Forster AJ, Taljaard M, Oake N, Wilson K, Roth V, van Walraven C (2012) The effect of hospital-acquired infection with Clostridium difficile on length of stay in hospital. CMAJ 184:37-42

3. van Kleef E, Green N, Goldenberg SD et al (2014) Excess length of stay and mortality due to Clostridium difficile infection: a multistate modelling approach. J Hosp Infect 88:213-217

4. Murphy CR, Avery TR, Dubberke ER, Huang SS (2012) Frequent hospital readmissions for Clostridium difficile infection and the impact on estimates of hospital-associated C. difficile burden. Infect Control Hosp Epidemiol 33:20-28

5. Zilberberg MD, Shorr AF, Micek ST, Kollef MH (2015) Clostridium difficile recurrence is a strong predictor of 30-day rehospitalization among patients in intensive care. Infect Control Hosp Epidemiol 36:273-279

6. Pépin J, Valiquette L, Cossette B (2005) Mortality attributable to nosocomial Clostridium difficile-associated disease during an epidemic caused by a hypervirulent strain in Quebec. CMAJ 173: 1037-1042

7. Rao K, Micic D, Natarajan M et al (2015) Clostridium difficile ribotype 027 : relationship to age, detectability of toxins A or B in stool with rapid testing, severe infection, and mortality. Clin Infect Dis 61:233-241

8. Olsen MA, Yan Y, Reske KA, Zilberberg MD, Dubberke ER (2015) Recurrent Clostridium difficile infection is associated with increased mortality. Clin Microbiol Infect 21:164-170

9. Deshpande A, Pasupuleti V, Thota P et al (2015) Risk factors for recurrent Clostridium difficile infection: a systematic review and meta-analysis. Infect Control Hosp Epidemiol 36:452-460

10. Abou Chakra CN, Pepin J, Sirard S, Valiquette L (2014) Risk factors for recurrence, complications and mortality in Clostridium difficile infection: a systematic review. PLoS One 9:e98400

11. Bauer MP, Kuijper EJ, van Dissel JT; European Society of Clinical Microbiology and Infectious Diseases (2009) European Society of Clinical Microbiology and Infectious Diseases (ESCMID): treatment guidance document for Clostridium difficile infection (CDI). Clin Microbiol Infect 15:1067-1079

12. Louie TJ, Miller MA, Mullane KM et al (2011) Fidaxomicin versus vancomycin for Clostridium difficile infection. N Engl J Med 364: 422-431

13. Cornely OA, Crook DW, Esposito R et al (2012) Fidaxomicin versus vancomycin for infection with Clostridium difficile in Europe, Canada, and the USA: a double-blind, non-inferiority, randomised controlled trial. Lancet Infect Dis 12:281-289

14. Department of Health (2011) Governance arrangements for research ethics committees: a harmonised edition (updated April 2012). Available online at: https://www.gov.uk/government/ publications/health-research-ethics-committees-governancearrangements. Accessed 24th June 2015

15. Kelly CP, LaMont JT (2008) Clostridium difficile - more difficult than ever. N Engl J Med 359:1932-1940

16. Johnson S (2009) Recurrent Clostridium difficile infection: causality and therapeutic approaches. Int J Antimicrob Agents 33:S33S36

17. Maguire PA, Taylor IC, Stout RW (1986) Elderly patients in acute medical wards: factors predicting length of stay in hospital. Br Med $\mathrm{J}$ (Clin Res Ed) 292:1251-1253

18. Campbell SE, Seymour DG, Primrose WR; ACMEPLUS Project (2004) A systematic literature review of factors affecting outcome in older medical patients admitted to hospital. Age Ageing 33:110 115

19. Jasinarachchi KH, Ibrahim IR, Keegan BC et al (2009) Delayed transfer of care from NHS secondary care to primary care in England: its determinants, effect on hospital bed days, prevalence of acute medical conditions and deaths during delay, in older adults aged 65 years and over. BMC Geriatr 9:4 